\title{
Double tracer gas single-breath washout: reproducibility in healthy subjects and COPD
}

\author{
Kim Husemann ${ }^{1}$, Nina Berg ${ }^{1}$, Jennifer Engel ${ }^{1}$, Johannes Port², \\ Christoph Joppek², Ziran Tao ${ }^{2}$, Florian Singer ${ }^{3}$, Holger Schulz ${ }^{4}$ and \\ Martin Kohlhäufl ${ }^{1}$
}

Affiliations: 'Klinik Schillerhöhe, Center for Respiratory Medicine and Thoracic Surgery, Robert-BoschKrankenhaus-GmbH, Stuttgart, Germany. ${ }^{2}$ University of Stuttgart, Dept of Biomedical Engineering, Stuttgart, Germany. ${ }^{3}$ University Children's Hospital Zurich, Switzerland. ${ }^{4 H e l m h o l t z ~ Z e n t r u m ~ M u n i c h, ~ I n s t i t u t e ~ o f ~}$ Epidemiology I, Neuherberg, Germany.

Correspondence: Kim Husemann, Klinik Schillerhöhe, Center for Respiratory Medicine and Thoracic Surgery, Robert-Bosch-Krankenhaus-GmbH, Solitudestr. 18, 70839 Gerlingen, Germany.

E-mail: kim.husemannaklinik-schillerhoehe.de

ABSTRACT The applicability and interpretation of inert tracer gas washout tests is hampered by the lack of feasible protocols and reproducibility data. We assessed feasibility, variability and reproducibility of a new easy to perform double tracer gas (DTG) single-breath washout (SBW) test and compared this with conventional nitrogen washouts.

In 40 healthy nonsmokers and 20 patients with stable chronic obstructive pulmonary disease (COPD), we performed three $\mathrm{N}_{2}$ vital capacity SBWs, three $\mathrm{N}_{2}$ multiple-breath washouts and three tidal DTG-SBW tests. Follow-up was after 1 week, 1 month and 6 months. Main outcomes were the lung clearance index (LCI) $\left(\mathrm{N}_{2}\right.$ multiple-breath washout), slope of phase III $\left(\mathrm{dN}_{2}\right)\left(\mathrm{N}_{2}\right.$ vital capacity SBW) and slope of phase III (SIIIDTG) (DTG-SBW).

In healthy subjects, mean \pm SD LCI at baseline was $6.94 \pm 0.61, \mathrm{dN}_{2} 0.99 \pm 0.42 \% \mathrm{~N}_{2}$ per litre and SIIIDTG $-0.206 \pm 0.108 \mathrm{~g} \cdot \mathrm{mol}^{-1} \cdot \mathrm{L}^{-1}$. In COPD, LCI and $\mathrm{dN}_{2}$ were significantly higher (LCI 12.23 $\pm 2.67, \mathrm{dN}_{2}$ $7.43 \pm 5.38 \% \mathrm{~N}_{2}$ per litre; $\left.\mathrm{p}<0.001\right)$ and SIIIDTG significantly steeper $\left(-0.653 \pm 0.428 \mathrm{~g} \cdot \mathrm{mol}^{-1} \cdot \mathrm{L}^{-1}, \mathrm{p}<0.001\right)$. Reproducibility was high for main outcome parameters: the intraclass correlation coefficient over 6 months was 0.77 (0.86 in COPD) for LCI, $0.82(0.89)$ for $\mathrm{dN}_{2}$ and 0.83 (0.93) for SIIIDTG.

The tidal DTG-SBW is a reproducible test in healthy and COPD subjects that seems attractive for use in routine clinical settings.

0

@ERSpublications

DTG-SBW may play a role in detection and monitoring of small airway diseases or as an outcome in drug trials http://ow.ly/x15bw

For editorial comments see page 1113 .

This article has supplementary material available from www.erj.ersjournals.com

Received: May 192013 | Accepted after revision: May 142014 | First published online: Aug 072014

Clinical trial: This study is registered in the German Clinical Trials Register with identifier number DRKS00003541.

Support statement: This study is part of an investigator-initiated trial supported in equal parts by grants from Chiesi GmbH (Hamburg, Germany) and the Robert Bosch Foundation (grant number KKF-12-8; Robert-Bosch Stiftung GmbH, Stuttgart, Germany).

Conflict of interest: Disclosures can be found alongside the online version of this article at www.erj.ersjournals.com

Copyright @ERS 2014 


\section{Introduction}

Inert tracer gas washout (IGW) tests are simple and noninvasive tools to detect air trapping and ventilation inhomogeneity due to patchy small airway obstruction [1]. The multiple-breath washout (MBW) technique and the vital capacity single-breath washout (SBW) technique are the two principal tests established. Compared with MBW, the vital capacity SBW protocol is considerably shorter but requires good coordination and cooperation to perform the vital capacity manoeuvre at constant flow. Until recently, the lack of commercially available hard- and software hampered the use of gas washout tests in research and in the clinical setting. Thus, the recent American Thoracic Society/European Respiratory Society consensus statement on IGW testing stressed the need for feasibility and reproducibility studies [2].

The new double tracer gas (DTG)-SBW test may be more feasible than other techniques due to its easy and fast application requiring only a few tidal breaths [3]. The test is based on a tidal in- and exhalation of a dual tracer gas mixture containing helium and sulphur hexafluoride $\left(\mathrm{He}_{\mathrm{SF}} 5 / 1\right)$. An ultrasonic flow meter (USFM) quantifies molar mass during in- and expiration, and accurately reflects $\mathrm{He}$ and $\mathrm{SF}_{6}$ washout patterns in the expired air [3].

The tracer gases $\mathrm{SF}_{6}$ and $\mathrm{He}$ have frequently been used separately, while recent data for the simultaneous use of a $\mathrm{SF}_{6}-\mathrm{He}$ dual tracer gas mixture in adults are rare [4-6]. The rationale behind using a dual tracer gas mixture is to assess specific ventilation inhomogeneity in distal airways. As the contribution of convectiondependent ventilation inhomogeneity for $\mathrm{He}$ and $\mathrm{SF}_{6}$ is supposed to be equal, convection-diffusiondependent ventilation inhomogeneity differs for $\mathrm{He}_{\text {and }} \mathrm{SF}_{6}$, and is supposed to arise in different areas of the pre-acinar and acinar lung zones. While the simultaneous analysis of $\mathrm{He}$ and $\mathrm{SF}_{6}$ washout (usually assessed as slope difference between $\mathrm{SF}_{6}$ and $\mathrm{He}$ in a $\mathrm{SBW}$ ) can be interpreted as a specific index for peripheral ventilation inhomogeneity, the comparable signal of a single tracer gas mainly provides information about overall ventilation inhomogeneity [7].

Parameters of the new tidal DTG-SBW were associated with global and acinar ventilation inhomogeneity indices in children with cystic fibrosis lung disease [8]. In this study, the tidal DTG-SBW was much faster on average (3 versus $30 \mathrm{~min}$ for three trials) and easier (success rate 92\% versus $82 \%$ ) [8]. Recent data suggest that the DTG-SBW may be a sensitive test to assess ventilation inhomogeneity in the lung periphery in different clinical conditions (e.g. detection of small airway dysfunction in children with mild asthma and normal spirometry [9] or improved gas mixing efficiency as an immediate treatment response to physiotherapy in children with mild cystic fibrosis lung disease [10]).

In the current study, we assessed the feasibility, variability and short/long-term reproducibility of the new tidal DTG-SBW-test in adult healthy nonsmokers and patients with stable chronic obstructive pulmonary disease (COPD). Classical and new outcome parameters of the test were determined, and their association with established $\mathrm{N}_{2}$ washout indices and conventional lung function parameters was assessed. Variability and reproducibility estimates of all IGW outcomes were compared. Secondary end-points were differences in ventilation inhomogeneity between groups.

\section{Materials and methods \\ Study design, study population and ethics}

This study is part of a larger prospective cohort study (Evaluation of $\mathrm{N}_{2}$ single- and multiple-breath washout versus a double-tracer single-breath technique in healthy adults and patients with small airway diseases; German Clinical Trials Register DRKS00003541). This trial was approved by the Ethics Committee of the Landesaerztekammer Baden-Wuerttemberg (AZ F-2011-073) and performed at the Klinik Schillerhoehe, Center for Respiratory Medicine and Thoracic Surgery, Robert-Bosch-Krankenhaus$\mathrm{GmbH}$, Stuttgart, Germany. 40 healthy nonsmokers and 20 patients with clinically stable COPD (spirometric grading Global Initiative for Chronic Obstructive Lung Disease (GOLD) 1-3) were recruited between April 2012 and August 2013 (table 1). COPD subjects had a clinical diagnosis of COPD according to GOLD with a post-bronchodilator forced expiratory volume in $1 \mathrm{~s}$ (FEV1)/forced vital capacity ratio $<0.7$ and a smoking history $\geqslant 10$ pack-years [11]. Patients with very severe airflow obstruction (FEV1 $<30 \%$ predicted), supplemental oxygen therapy or recent exacerbation were excluded. After written informed consent was obtained, all participants performed a routine lung function test (spirometry and body plethysmography); COPD subjects had an additional baseline measurement of single-breath diffusing capacity of the lung for carbon monoxide (DLCO). Subsequently, we performed three $\mathrm{N}_{2} \mathrm{MBW}$, three $\mathrm{N}_{2}$ vital capacity SBW and three DTG-SBW tests. Follow-up was after 1 week, 1 month and 6 months. Each visit included the same lung function tests. COPD patients continued their regular bronchodilator medication before each visit. 
TABLE 1 Clinical and demographical characteristics of healthy and chronic obstructive pulmonary disease (COPD) subjects

\begin{tabular}{|c|c|c|c|}
\hline & Healthy & COPD & p-value \\
\hline Subjects & 40 & 20 & \\
\hline Age years & $40.7 \pm 10.3$ & $66.2 \pm 10.3$ & $<0.001$ \\
\hline Males/females & $12: 28$ & $16: 04$ & \\
\hline Weight kg & $69.0 \pm 13.8$ & $79.1 \pm 16.7$ & $<0.05$ \\
\hline Height cm & $169 \pm 8$ & $173 \pm 9$ & 0.068 \\
\hline BMI $\mathrm{kg} \cdot \mathrm{m}^{-2}$ & $24.1 \pm 3.50$ & $26.3 \pm 4.1$ & $<0.05$ \\
\hline FVC $\%$ pred & $102.2 \pm 12.1$ & $83.7 \pm 13.9$ & $<0.001$ \\
\hline Z-score & $0.18 \pm 0.99$ & $-0.93 \pm 0.91$ & $<0.001$ \\
\hline FEV $1 \%$ pred & $101.0 \pm 11.5$ & $55.2 \pm 13.9$ & $<0.001$ \\
\hline Z-score & $0.06 \pm 0.92$ & $-2.60 \pm 0.80$ & $<0.001$ \\
\hline MMEF \% pred & $97.2 \pm 18.2$ & $26.6 \pm 11.0$ & $<0.001$ \\
\hline Z-score & $-0.14 \pm 0.69$ & $-2.46 \pm 0.64$ & $<0.001$ \\
\hline DLco $\%$ pred & ND & $57.5 \pm 19.4$ & \\
\hline$F_{R C} C_{\text {MBW }} \mathrm{L}$ & $3.21 \pm 0.81$ & $4.19 \pm 1.27$ & $<0.05$ \\
\hline $\mathrm{LCl}$ & $6.94 \pm 0.61$ & $12.23 \pm 2.67$ & $<0.001$ \\
\hline Scond $\mathrm{L}^{-1}$ & $0.028 \pm 0.026$ & $0.045 \pm 0.034$ & $<0.05$ \\
\hline Scond $\times V_{T}$ & $0.029 \pm 0.029$ & $0.046 \pm 0.034$ & 0.061 \\
\hline Sacin $\mathrm{L}^{-1}$ & $0.058 \pm 0.028$ & $0.343 \pm 0.139$ & $<0.001$ \\
\hline Sacin $\times V T$ & $0.060 \pm 0.034$ & $0.355 \pm 0.150$ & $<0.001$ \\
\hline $\mathrm{dN}_{2} \% \mathrm{~N}_{2}$ per $\mathrm{L}$ & $0.99 \pm 0.42$ & $7.43 \pm 5.38$ & $<0.001$ \\
\hline $\mathrm{dN}_{2} \times \mathrm{VC} \% \mathrm{~N}_{2}$ & $3.82 \pm 1.36$ & $20.60 \pm 6.84$ & $<0.001$ \\
\hline SIIIDTG $\mathrm{g} \cdot \mathrm{mol}^{-1} \cdot \mathrm{L}^{-1}$ & $-0.206 \pm 0.108$ & $-0.653 \pm 0.428$ & $<0.001$ \\
\hline SIIIDTG $\times$ VT $\mathbf{g} \cdot \mathrm{mol}^{-1}$ & $-0.204 \pm 0.091$ & $-0.635 \pm 0.344$ & $<0.001$ \\
\hline AUC $\mathrm{g} \cdot \% \cdot \mathrm{mol}^{-1}$ & $16.39 \pm 5.37$ & $33.04 \pm 18.59$ & $<0.001$ \\
\hline AUC $60-90 \mathrm{~g} \cdot \% \cdot \mathrm{mol}^{-1}$ & $2.69 \pm 1.52$ & $6.62 \pm 4.92$ & $<0.05$ \\
\hline Peak g $\cdot \mathrm{mol}^{-1}$ & $0.59 \pm 0.15$ & $0.77 \pm 0.30$ & $<0.001$ \\
\hline Peak volume \% & $19.00 \pm 3.08$ & $23.86 \pm 5.09$ & $<0.001$ \\
\hline FWHM \% & $19.54 \pm 7.82$ & $36.71 \pm 11.64$ & $<0.001$ \\
\hline
\end{tabular}

Data are presented as $\mathrm{n}$ or mean \pm SD. $\mathrm{p}$-values derived from two-sample t-test. BMI: body mass index; FVC: forced vital capacity; FEV1: forced expiratory volume in $1 \mathrm{~s}$; MMEF: maximal midexpiratory flow; DLCO: diffusing capacity of the lung for carbon monoxide; $\mathrm{FRC}_{\mathrm{MBW}}$ : functional residual capacity measured by nitrogen multiple-breath washout; LCl: lung clearance index; Scond: index of conductive ventilation inhomogeneity; $V_{T}$ : tidal volume; Sacin: index of acinar ventilation inhomogeneity; $\mathrm{dN}_{2}$ : phase III slope of nitrogen single-breath washout; VC: vital capacity; SIIIDTG: phase III slope of double tracer gas single-breath washout; AUC: area under the curve for double tracer gas single-breathe washout; AUC60-90: area under the curve between 60\% and $90 \%$ of expired volume for double tracer gas single-breath washout; peak: absolute peak concentration of the double tracer gas single-breath washout signal; peak volume: proportion of exhaled volume at peak; FWHM: full width at half maximum of double tracer gas single-breath washout; ND: not done.

\section{Lung function procedures}

Spirometry, body plethysmography and single-breath DLCO

We performed routine lung function testing with standard laboratory equipment (JAEGER MasterScreen; CareFusion, Hoechberg, Germany) as recommended by current standards [12-14]. For all spirometric parameters, we used the recently published reference values of the Global Lungs Initiative [15]. Predicted values for DLCO were derived from those of COTES et al. [16].

\section{$\mathrm{N}_{2}$ vital capacity SBW and $\mathrm{N}_{2} \mathrm{MBW}$}

The classical $\mathrm{N}_{2}$ vital capacity SBW and the $\mathrm{N}_{2}$ MBW were performed using the commercially available device Exhalyzer D (Eco Medics AG, Duernten, Switzerland) according to recommendations [2]. Primary outcome parameters were the slope of phase III $\left(\mathrm{dN}_{2}\right)$ for $\mathrm{N}_{2}$ vital capacity SBW, and functional residual capacity (FRC) and lung clearance index (LCI) for $\mathrm{N}_{2}$ MBW. We additionally calculated MBW indices of conductive (Scond) and acinar (Sacin) ventilation inhomogeneity originally described by VERBANCK et al. [17]. All SBW and MBW parameters were computed with the software provided with the Exhalyzer D (Spiroware version 3.1.6; Eco Medics AG). We applied volume correction to all slope III estimates to account for physiological variations in tidal volume $(V \mathrm{~T}): \mathrm{dN}_{2} \times$ expiratory vital capacity, Scond $\times V \mathrm{~T}$ and Sacin $\times V T$, 


\section{Double tracer gas single-breath washout}

The tidal DTG-SBW was performed with the Exhalyzer D and corresponding software for data recording. Setup, measurement and analysis were initially described by SINGER et al. [3]. The DTG mixture containing oxygen $(21.0 \%), \mathrm{N}_{2}(47.7 \%), \mathrm{He}(26.3 \%)$ and $\mathrm{SF}_{6}(5.0 \%)$ had a similar molar mass to dry medical air $\left(28.9 \mathrm{~g} \cdot \mathrm{mol}^{-1}\right)$, and was provided by Westfalen-AG (Muenster, Germany). During regular quiet breathing, the DTG was applied for a single tidal inhalation from FRC. In the pre-test phase, during the DTG in/ expiration and the following inspiration, we recorded molar mass with a side-stream USFM, tidal flows with a mainstream USFM, and carbon dioxide and $\mathrm{O}_{2}$ signals (fig. 1a).

The molar mass signal measured in side-stream mode (MMss) during ambient air breathing was used to calibrate the pre-test $\mathrm{CO}_{2}$ signal, i.e. to transform the pre-test $\mathrm{CO}_{2}$ ignal by linear regression modelling into an additional molar mass signal (calculated molar mass signal (MMcalc); for details, see the online supplementary material). By "subtracting" MMcalc from the raw MMss during expiration of the DTG, the molar mass test signal reflecting the agglomerated $\mathrm{SF}_{6}$ and $\mathrm{He}$ washout pattern was obtained (fig. $1 \mathrm{a}$ and $\mathrm{b}$ ) [3]. The DTG expirogram (MMss-MMcalc) was plotted against expired volume (fig. 1c). The main outcome parameter was the phase III slope between $60 \%$ and $90 \%$ of the expired volume (SIIIDTG), which was determined by automated linear regression. SIIIDTG probably detects gas mixing efficiency near the acinar lung regions (diffusion-dependent ventilation inhomogeneity) $[8,9]$. To account for variations in tidal volume $(V \mathrm{~T})$, we also performed a volume correction for SIIIDTG (SIIIDTG $\times V \mathrm{~T}$ ) as proposed previously $[8,18]$.

We described visible dynamics of the simultaneous $\mathrm{He}_{\mathrm{SF}}$ washout signal by additional outcome parameters, tracking differences in the shape of the curve between healthy and COPD subjects. As an additional phase III parameter, we calculated the area under the curve between $60 \%$ and $90 \%$ of the expired volume (AUC60-90). Parameters from tidal phase II were the absolute peak concentration of the signal (peak), the peak volume (percentage of exhaled volume at peak) and the area under the curve over the total expired volume (AUC). To describe the skewness of the DTG signal, we measured the full width at half maximum (FWHM). All DTG-SBW data analysis was performed with in-house customised software based on LabVIEW 2012 (National Instruments, Austin, TX, USA).

A detailed description of the equipment, procedures and analysis for all washout procedures, including an illustration of all outcome parameters for DTG-SBW, is provided in the online supplementary material.

\section{Statistical analysis}

Demographic data such as age, weight and height are reported as mean, standard deviation and range. Individual data for all IGW parameters were reported as mean and intra-individual standard deviation of three technically acceptable tests. Parameters between groups were compared using two-sample-t-tests.

a)

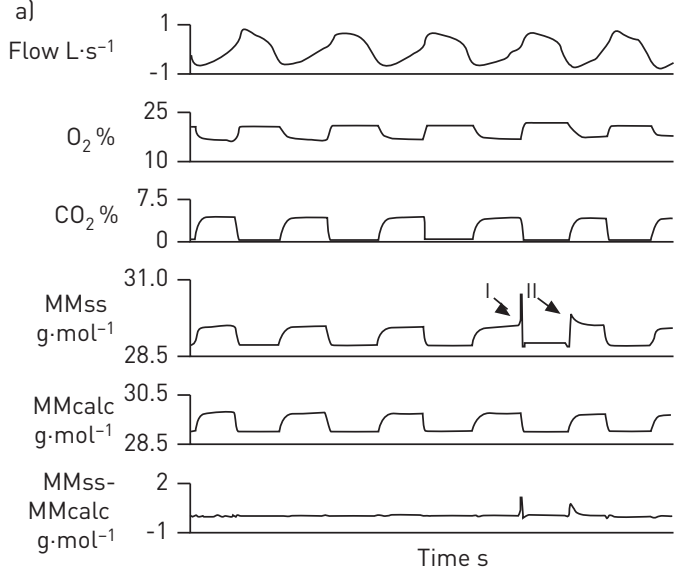

b)
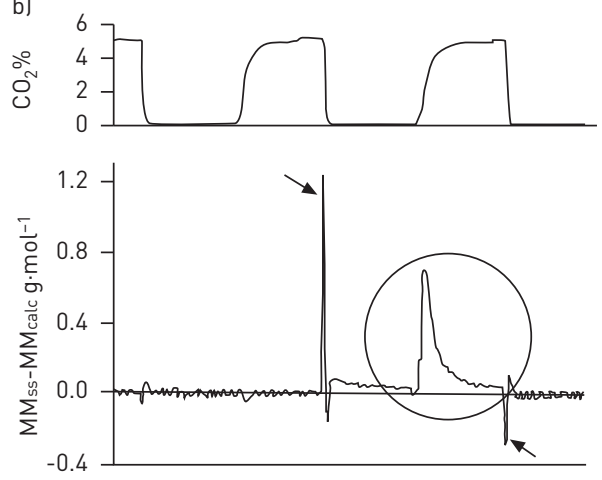

Time s c)

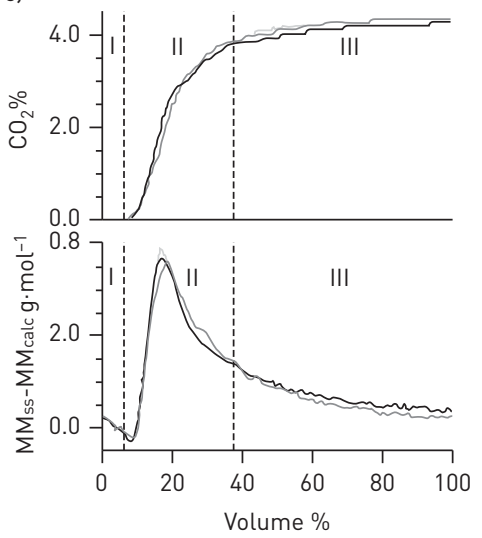

FIGURE 1 Synchronised raw data and dual tracer gas (DTG) test signal. a) Synchronised raw data of DTG single-breath washout. Flow (measured as body temperature, pressure and saturation), oxygen concentration, carbon dioxide concentration, molar mass signal measured in side-stream mode (MMss), calculated molar mass signal (MMcalc) and difference signal (MMss-MMcalc). I: start of inspiration of DTG; II: the following expiration shows a different MMss signal compared with the naturally exhaled MMss signal in the previous breaths. b) DTG test signal. $\mathrm{CO}_{2}$ signal and MMss-MMcalc corresponding to the final DTG test signal (circled). Small artefacts in MMss-MMcalc between in- and expiration are due to gas and sensor dynamics during flow inversion. At the moment of inspiration of the DTG (upper arrow) and the subsequent inspiration (lower arrow), there are two spikes explained by gas de-mixing phenomena due to the technical set-up. c) Typical DTG signal of a healthy adult. Three expirograms of $\mathrm{CO}_{2}$ and $\mathrm{MMss}_{\mathrm{M}} \mathrm{M}$ calc on one test occasion. $\mathrm{CO}_{2}$ and $\mathrm{MMss}-\mathrm{MMcalc}$ were plotted against expired volume (\% of total expired volume). Phase I: dead space; phase II: transitional phase; phase III: alveolar phase. 
Intra-visit reproducibility was assessed as distribution (mean $\pm \mathrm{SD}$ ) of the intraindividual coefficients of variation of three trials (intraindividual sD/mean). We calculated the intra-class correlation coefficient (ICC) in a two-way mixed model to assess intra-visit reproducibility (three individual trials at one test occasion) as well as inter-visit reproducibility (baseline, 1 week, 1 month and 6 months). The ICC compares within-subject variance to total variance across all measurements and subjects, and is appropriate to compare repeatability on untransformed data measured on different scales [19]. Differences between visits were assessed graphically by the method described by BLAND and ALTMAN [20], and quantified by the coefficient of repeatability representing a $95 \%$ range of differences between two measurements. The coefficient of repeatability was calculated as $1.96 \times \mathrm{SD}$ of the differences between two measurements [20].

To describe the association between IGW indices and age and conventional lung function parameters, we performed simple linear regression.

To assess effects of breathing pattern and lung volume on DTG-SBW indices, we used a linear mixed-effects model with a random intercept to account for multiple observations. Variance components of random and fixed effects were assessed with the residual maximum likelihood method. All statistical analyses were performed using JMP (version 10.0; SAS Institute, Cary, NC, USA) for Windows and a p-value of 0.05 or less was regarded as significant.

\section{Results}

40 healthy nonsmokers (aged 20-64 years) and 20 patients with stable COPD (aged 53-83 years, FEV1 31$78 \%$ predicted) were recruited. The clinical and demographic baseline characteristics of both groups are outlined in table 1.38 out of 40 healthy subjects ( 15 out of 20 COPD patients) completed the whole study. The test success rate (three technically acceptable tests at one occasion) was $88 \%$ for $\mathrm{N}_{2} \mathrm{MBW}$ (75\% in COPD), 95\% for $\mathrm{N}_{2}$ vital capacity SBW (99\% in COPD) and 97\% for DTG-SBW (96\% in COPD).

In the COPD group, indices of $\mathrm{N}_{2}$ vital capacity SBW and $\mathrm{N}_{2} \mathrm{MBW}$ were significantly different from healthy adults while $S$ cond $\times V$ T was of borderline significance $(0.029$ versus $0.046, \mathrm{p}=0.061)$ (table 1$)$. All outcome parameters describing the shape of the DTG-SBW curve (SIIIDTG $(\times V \mathrm{~T})$, AUC, AUC60-90, peak, peak volume and FWHM) were significantly different between both groups. The difference between SIIIDTG and SIIIDTG $\times V$ T was small (mean $\pm \mathrm{SD}$ difference $0.002 \pm 0.049 \mathrm{~g} \cdot \mathrm{mol}^{-1} \cdot \mathrm{L}^{-1}\left(95 \% \mathrm{CI}-0.018-0.014 \mathrm{~g} \cdot \mathrm{mol}^{-1} \cdot \mathrm{L}^{-1}\right)$ in healthy subjects; $0.018 \pm 0.146 \mathrm{~g} \cdot \mathrm{mol}^{-1} \cdot \mathrm{L}^{-1}\left(95 \% \mathrm{CI}-0.094-0.059 \mathrm{~g} \cdot \mathrm{mol}^{-1} \cdot \mathrm{L}^{-1}\right)$ in COPD).

In healthy subjects, we found an age-dependent increase in LCI $\left(\mathrm{r}^{2}=0.34, \mathrm{p}<0.001 ; \mathrm{LCI}=5.529+0.0345 \times\right.$ age $)$, and a marginal association between age and AUC $\left(\mathrm{r}^{2}=0.11, \mathrm{p}<0.05 ; \mathrm{AUC}=9.39+0.172 \times\right.$ age $)$. All other washout parameters did not correlate with age (online supplementary fig. 3 ).

The influence of tidal volume, flow and FRC on DTG-SBW main outcome parameters was assessed by multiple linear regression analysis in a mixed-effects model obtained across all subjects (with different breathing manoeuvres) (table 2 and online supplementary fig. 5). The breathing pattern ( $V \mathrm{~T}$ and flow) was comparable in healthy and COPD subjects (mean $\pm \mathrm{SD} V \mathrm{~T} 1.05 \pm 0.22$ versus $1.05 \pm 0.21 \mathrm{~L}$; expiratory flow $-0.65 \pm 0.19$ versus $\left.-0.69 \pm 0.17 \mathrm{~L} \cdot \mathrm{s}^{-1}\right)$. According to our model, $69-81 \%$ of the total variance can be explained by clustering within healthy subjects (92-97\% in COPD). Thus, only a minor proportion of the total variance can be attributed to the breathing manoeuvre itself. However, extreme values of $V \mathrm{~T}$, flow or FRC may have considerable influence on DTG-SBW parameters (e.g. change of $1 \mathrm{~L}$ in $V \mathrm{~T}$ leads to change of $0.262 \mathrm{~g} \cdot \mathrm{mol}^{-1} \cdot \mathrm{L}^{-1}$ in SIIIDTG in healthy subjects).

Typical DTG-SBW signals of COPD patients are shown in figure 2. SIIIDTG is steeper and AUC is higher compared with the signal in healthy nonsmokers (fig. 2 versus fig. 1c). Patient B in figure 2, with lower DLCO, presumably due to a higher extent of emphysema, shows a steeper slope (SIIIDTG) and a greater AUC than patient A despite their comparable airflow limitation (FEV1 40\% pred). In the COPD group, DLCO as surrogate marker of emphysema extent correlates with $\operatorname{Sacin} \times V \mathrm{~T}\left(\mathrm{r}^{2}=0.41, \mathrm{p}<0.01\right), \mathrm{dN}_{2} \quad\left(\mathrm{r}^{2}=0.21\right.$, $\mathrm{p}<0.05)$ and main DTG-SBW indices (SIIIDTG $(\times V \mathrm{~T}): \mathrm{r}^{2}=0.33(0.31), \mathrm{p}<0.05$; AUC: $\left.\mathrm{r}^{2}=0.22, \mathrm{p}<0.05\right)$. FEV1 as marker of airway obstruction shows no association to SIIIDTG $(\times V \mathrm{~T})$ or other DTG-SBW indices (fig. 3; complete data including associations between DTG-SBW indices and $\mathrm{N}_{2}$ washout indices in online supplementary tables 2 and 3 ).

\section{Variability and reproducibility}

The mean intra-visit coefficient of variation in healthy subjects was $4.4 \%$ for LCI (5.3\% in COPD), $16.4 \%$ for $\mathrm{dN}_{2}(7.8 \%$ in COPD) and $22.2 \%$ for SIIIDTG $(10.1 \%$ in COPD) (table 3$)$. Scond $(\times V \mathrm{~T})$ and Sacin $(\times V \mathrm{~T})$ showed a clearly higher coefficient of variation $(72.9 \%$ (73.1\%) and 48.7\% (44.4\%), respectively). In COPD, with slightly higher absolute values for $S_{\text {cond }}(\times V \mathrm{~T})$ and considerably higher values for $\operatorname{Sacin}(\times V \mathrm{~T})$, the mean coefficient of variation was $45.1 \%(29.8 \%)$ and $16.3 \%(8.8 \%)$, respectively. For additional DTG-SBW 
TABLE 2 Effect of breathing pattern and lung volume on dual tracer gas single-breath washout parameters

\begin{tabular}{|c|c|c|c|}
\hline & SIIIDTG $\mathrm{g} \cdot \mathrm{mol}^{-1} \cdot \mathrm{L}^{-1}$ & AUC $\mathrm{g} \cdot \% \cdot \mathrm{mol}^{-1}$ & Peak $\mathbf{g} \cdot \mathrm{mol}^{-1}$ \\
\hline \multicolumn{4}{|l|}{ Healthy adults } \\
\hline Flow $L \cdot s^{-1}$ & $0.133(0.056-0.210)^{* * *}$ & $10.43(5.71-15.15)^{* * *}$ & $-0.49(-0.65-0.33)^{* * *}$ \\
\hline$V T \mathrm{~L}$ & $0.262(0.181-0.345)^{* * *}$ & $-6.15(-15.81--8.26)^{* * *}$ & $-0.15(-0.31-0.01)$ \\
\hline FRC L & $-0.028(-0.049--0.007)^{*}$ & $1.69(0.63-2.74)^{*}$ & $0.06(0.02-0.10)^{*}$ \\
\hline Constant & -0.307 & 10.43 & 0.25 \\
\hline Intraclass correlation & 0.81 & 0.87 & 0.69 \\
\hline $\mathrm{R}^{2}$ & 0.89 & 0.91 & 0.79 \\
\hline \multicolumn{4}{|l|}{ COPD patients } \\
\hline Flow $L \cdot S^{-1}$ & $-0.228(-0.543-0.088)$ & $0.79(-9.27-10.85)$ & $0.03(-0.20-0.26)$ \\
\hline VT L & $0.494(0.145-0.842) *$ & $-2.84(-14.16-8.47)$ & $-0.04(-0.30-0.22)$ \\
\hline FRC L & $-0.037(-0.101-0.028)$ & $1.65(-0.44-3.74)$ & $0.05(0.00-0.09)$ \\
\hline Constant & -1.203 & 29.90 & 0.66 \\
\hline Intraclass correlation & 0.92 & 0.97 & 0.94 \\
\hline $\mathrm{R}^{2}$ & 0.96 & 0.98 & 0.96 \\
\hline \multicolumn{4}{|c|}{ 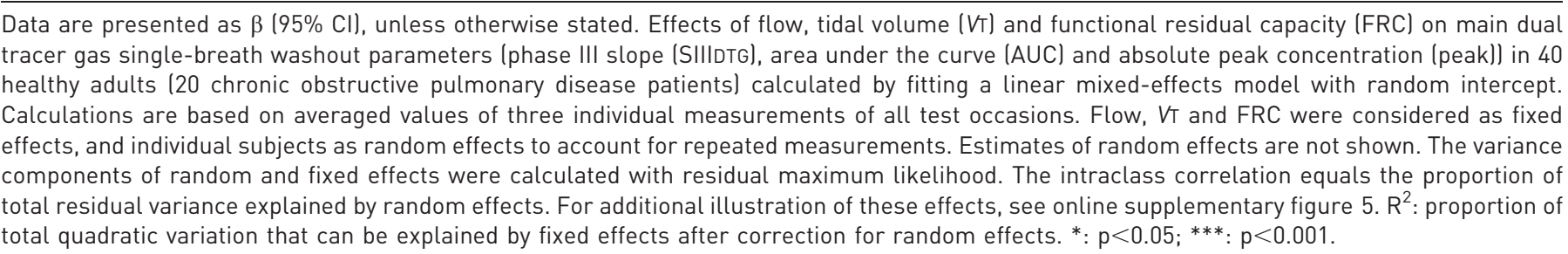 } \\
\hline
\end{tabular}

parameters, the mean intra-visit coefficient of variation was between $7 \%$ and $18 \%$ in both groups (data not shown).

The intra-visit ICC was $>0.7$ for all gas washout parameters except $\operatorname{Sacin}(\times V \mathrm{~T})$ in healthy adults $($ ICC 0.42 $(0.52))$ and $S$ cond $(\times V T)$ in both groups (ICC $0.44(0.62)$ in healthy subjects and $0.67(0.66)$ in COPD). The intra-visit ICC was comparable for uncorrected and "volume"-corrected phase III slope indices in both groups (table 3).

The mean difference between tests was small for all three washouts, considering short-term as well as longterm reproducibility (table 4, fig. 4 and online supplementary fig. 6). The inter-visit ICC was $>0.75$ for all washout parameters except MBW slope III indices $(S$ cond $(\times V T)$ and Sacin $(\times V T)$ ) in healthy subjects and Scond $(x V T)$ in COPD. Inter-visit variability was comparable for uncorrected and volume-corrected phase III slope indices.

In healthy adults, the coefficient of repeatability ranged between 0.83 and 0.96 for LCI (corresponding to $11.9-13.8 \%$ of mean LCI). The coefficient of repeatability for $\mathrm{dN}_{2}$ was between $0.39 \%$ and $0.89 \% \mathrm{~N}_{2}$ per $\mathrm{L}$ (39.6-89.5\% of mean $\left.\mathrm{dN}_{2}\right)$, and for SIIIDTG between 0.098 and $0.140 \mathrm{~g} \cdot \mathrm{mol}^{-1} \cdot \mathrm{L}^{-1}(47.6-67.8 \%$ of mean SIIIDTG). In COPD, the absolute coefficient of repeatability was higher for all washout parameters except for Scond $(\times V \mathrm{~T})($ table 3$)$.

\section{Discussion}

This is the first study to analyse variability and long-term reproducibility of easily obtained measures of ventilation inhomogeneity based on commercially available hardware, the new tidal DTG-SBW, in adults. Our results show that the DTG-SBW is a feasible and reproducible test in subjects with and without COPD. It is easy to perform, with a success rate of $\geqslant 96 \%$. However, analysis has to be performed offline using custom software.

\section{Washout indices in healthy subjects}

Parameters of conventional lung function and $\mathrm{N}_{2}$ vital capacity SBW in healthy subjects are in accordance with previously published normative data [15, 21-23]. All $\mathrm{N}_{2} \mathrm{MBW}$ indices corresponded well to preliminary reference values obtained with the same equipment and protocol in adults [24]. A small, linear age-dependent increase in $\mathrm{N}_{2}$ MBW parameters (LCI, Scond and Sacin) was described before with the strongest association between age and LCI [25]. We confirmed a comparable rise in LCI (about 0.3 per 10 years). The possibly low sensitivity of our study to detect the age dependency of Scond and Sacin may be attributed to the smaller sample size $(n=40$ versus $n=120[25])$ with few individuals aged $>55$ years. Since 

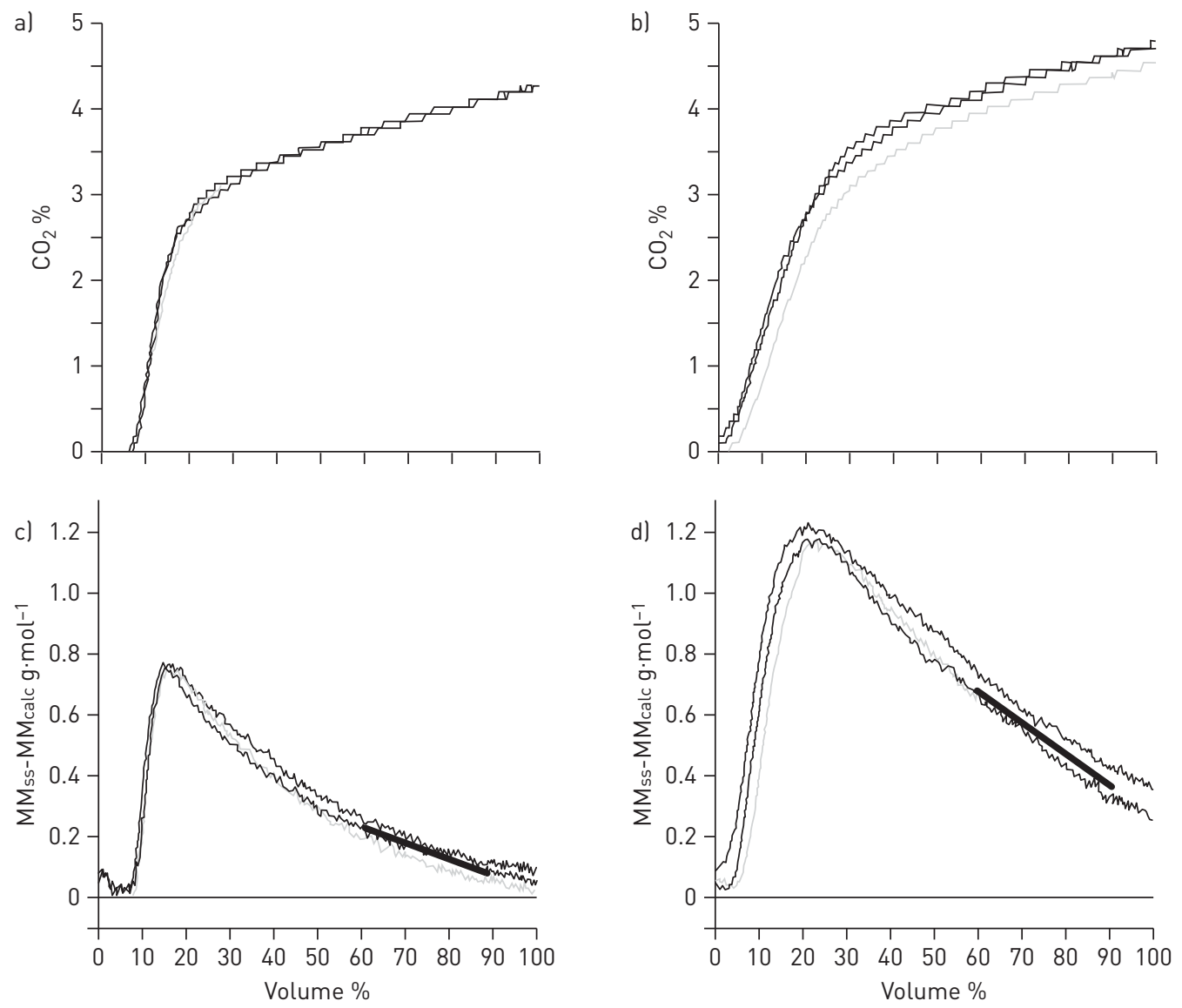

FIGURE 2 Typical dual tracer gas (DTG) single-breath washout (SBW) signals in chronic obstructive pulmonary disease (COPD). DTG-SBW signal in two COPD patients with comparable airflow limitation (postbronchodilator forced expiratory volume in $1 \mathrm{~s} 40 \%$ predicted) and different diffusing capacity of the lung for carbon monoxide (DLCO) as a noninvasive marker for emphysema extent. An expirogram of a, b) carbon dioxide and c, d) difference signal (MMssMMcalc) at one test occasion (three trials) plotted against expired volume (\% of total expired volume). a, c) Patient A: 73 years old, male, DLCO 63\% predicted. b, d) Patient B: 65 years old, male, DLCO 42\% predicted. Parameters of nitrogen vital capacity SBW and multiple-breath washout were comparable in both patients (phase III slope 5.0 versus $6.2 \% \cdot \mathrm{L}^{-1}$; lung clearance index 13.7 versus 12.7; index of conductive ventilation inhomogeneity $\times$ tidal volume (VT) 0.073 versus 0.068 ; index of acinar ventilation inhomogeneity $\times V$ T 0.447 versus 0.443 ). However, the phase III slope of DTG-SBW signal between $60 \%$ and $90 \%$ of expired volume (SIIIDTG; black solid line) was markedly steeper ( -0.404 versus $\left.-1.352 \mathrm{~g} \cdot \mathrm{mol}^{-1} \cdot \mathrm{L}^{-1}\right)$, and absolute peak concentration of the DTG-SBW signal and area under the curve of the DTG-SBW signal clearly greater $\left(0.75\right.$ versus $1.17 \mathrm{~g} \cdot \mathrm{mol}^{-1}$ and 29 versus $63 \mathrm{~g} \cdot \% \cdot \mathrm{mol}^{-1}$, respectively) in patient B.

only a marginal association between AUC (DTG-SBW) and age was detectable, we suggest the association between age and DTG-SBW indices be verified in a larger series.

\section{Washout indices in COPD}

This is the first study, using commercially available equipment, to show that in COPD with moderate-tosevere airflow limitation, classical measures of global and acinar ventilation inhomogeneity are significantly elevated. This is in accordance with previously described washout data in COPD [26-29]. The DTG-SBW signal markedly differs from the signal in healthy nonsmokers but seems comparable to the pattern observed in children with cystic fibrosis lung disease [8]. In these patients, SIIIDTG was significantly steeper than in healthy controls $\left(-0.457\right.$ versus $\left.-0.088 \mathrm{~g} \cdot \mathrm{mol}^{-1} \cdot \mathrm{L}^{-1}\right)$, and correlated with LCI and Sacin as marker of global and acinar ventilation inhomogeneity [8]. In COPD subjects, we observed a comparably steep slope III (SIIIDTG -0.653 versus $-0.206 \mathrm{~g} \cdot \mathrm{mol}^{-1} \cdot \mathrm{L}^{-1}$ in healthy) and a greater AUC (reflecting greater $\mathrm{SF}_{6}$ contribution to the signal). Both parameters were associated with DLCO as marker of peripheral airway disease and emphysema extent. SIIITG $\times V T$ and AUC were correlated with Sacin as index of acinar ventilation inhomogeneity, but not with global markers of ventilation inhomogeneity $\left(\mathrm{dN}_{2}\right.$ and LCI; online supplementary material). In a former study using mass spectrometry for simultaneous $\mathrm{He}_{2} \mathrm{SF}_{6}$ washout in 27 adult smokers, the $\mathrm{SF}_{6}-\mathrm{He}$ slope difference could differentiate between subjects with/without 

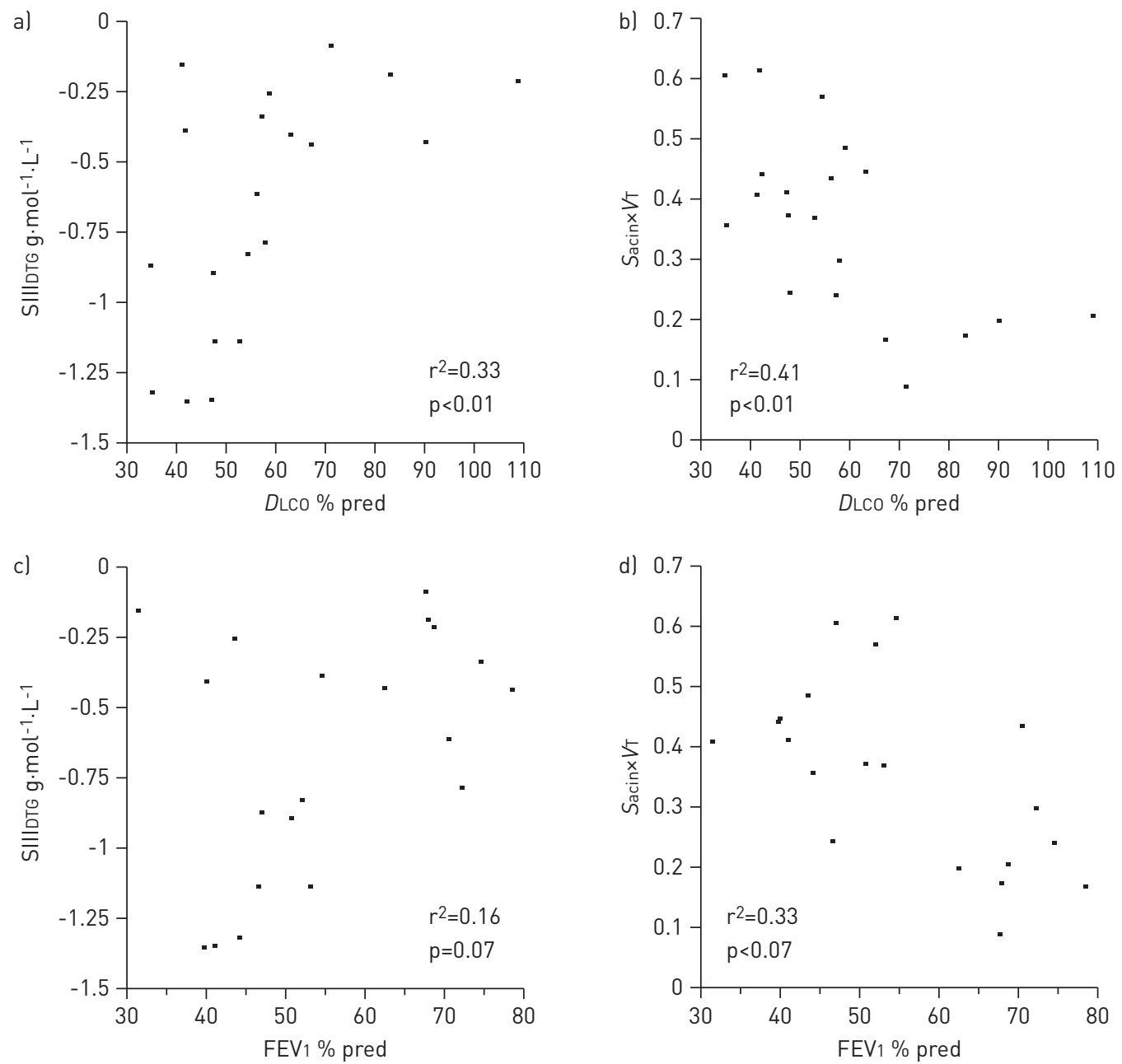

FIGURE 3 Association between phase III slope of double-tracer gas single-breath washout signal (SIIIDTG) and index of acinar ventilation inhomogeneity $(S a c i n) \times$ tidal volume $(V T)$ and conventional lung function in chronic obstructive pulmonary disease. Baseline a, c) SIIIDTG and b, d) Sacin $\times V$ T are plotted against a, b) diffusing capacity of the lung for carbon monoxide (DLCO) and c, d) forced expiratory volume in $1 \mathrm{~s}(\mathrm{FEV} 1) . \mathrm{r}^{2}$ : coefficient of determination.

histologically confirmed emphysema during one of several tidal breathing manoeuvres $(\mathrm{FRC}+V \mathrm{~T}=1.5 \mathrm{~L}$ ) [5]. In contrast, the global ventilation inhomogeneity index $\mathrm{N}_{2}$ slope (classical $\mathrm{N}_{2}$ vital capacity SBW) was not significantly different in subjects with/without emphysema [5].

In the context of previous clinical DTG-SBW data and former studies on simultaneous $\mathrm{He} / \mathrm{SF}_{6}$ washout $[3,5,8,30]$, we assume that the main outcome parameters of the DTG-SBW (SIIIDTG and AUC) probably reflect structural acinar changes in our COPD patients.

\section{Reproducibility of washout indices}

LCI showed a low intra-visit variability assessed by the coefficient of variation (mean $<5 \%$ in healthy adults) in accordance with other published data [24,31]. The intra-visit coefficient of variation of DTGSBW indices ranged between $7 \%$ and $23 \%$ (22.2\% for SIIIDTG). In healthy children and children with cystic fibrosis lung disease, the intra-test variability of SIIIDTG was $18.5 \%$ and $23.9 \%$, respectively, consistent with our data [8]. In contrast, phase III parameters of $\mathrm{N}_{2} \mathrm{MBW}$ (Scond and Sacin) showed a clearly higher intravisit coefficient of variation. Particularly in healthy adults with values near zero, small variations in phase III slope lead to high coefficients of variation. In COPD, the coefficient of variation of phase III indices is considerably lower due to higher absolute values. This phenomenon was also observed by HORSLEY et al. [32]: the coefficient of variation of intra-visit repeats of $S$ cond $\times V T$ was lower in cystic fibrosis than in healthy volunteers $(32.7 \%$ versus $59.5 \%)$ and overall variability of phase III slope indices was consistent with our data. To better compare variability of our untransformed data on different scales, we also assessed the ICC. Intra-visit reproducibility measured by ICC was good $(>0.7)$ for the majority of washout parameters 
TABLE 3 Main gas washout parameters and intra-visit reproducibility

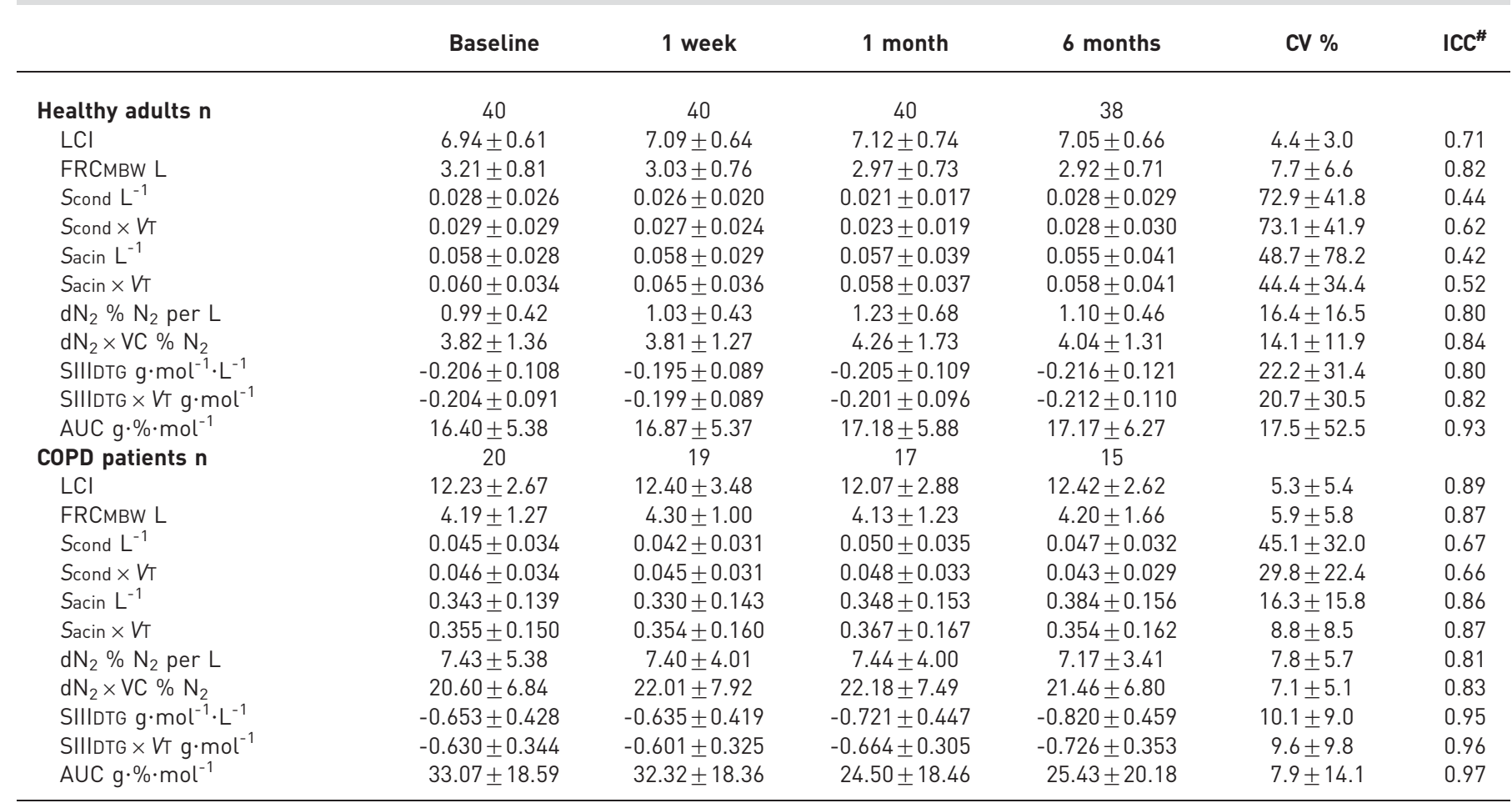

Data are presented as mean \pm SD unless otherwise stated. Intra-visit reproducibility was assessed as distribution (mean \pm SD) of the intraindividual coefficient of variation (CV) of three single measurements of all subjects and all test occasions. ICC: intraclass correlation coefficient; LCI: lung clearance index; FRCMBW: functional residual capacity measured by nitrogen multiple-breath washout; Scond: index of conductive ventilation inhomogeneity; VT: tidal volume; Sacin: index of acinar ventilation inhomogeneity; $\mathrm{dN}_{2}$ : phase III slope of nitrogen single-breath washout; VC: vital capacity; SIIIDTG: phase III slope of double tracer gas single-breath washout; AUC: area under the curve (double tracer gas single-breath washout). \#: calculated from three individual trials on the same day.

in both groups, except for phase III slope indices of MBW in healthy adults and $S$ cond $(\times V T)$ in COPD patients (ICC $0.42-0.66$ ). A recent study similarly found a poor intra-visit reproducibility of Scond (ICC 0.23 ) in 18 patients with moderate-to-severe asthma [33].

Over a period of 6 months, the long-term reproducibility of most washout indices was good (ICC $>0.75$ ), except for $S$ cond $(x V \mathrm{~T})$ in both groups and for $S$ acin $(x V \mathrm{~T})$ in healthy adults. Others reported comparable reproducibility of LCI (ICC $0.70-0.91$ ), but better reproducibility of Sacin and Scond (ICC 0.78-0.95) over a period of 8 days in 21 subjects [34]. In contrast to our study, the MBW was performed with a fixed 1-L breathing protocol, which may partly explain their lesser variability in phase III slope indices.

Intra- and inter-visit reproducibility of all DTG-SBW indices was high in both groups. The better reproducibility of SIIIDTG compared with Scond and Sacin in our study is somewhat counterintuitive, as the latter estimates are derived from slope values throughout a MBW procedure, whereas DTG-SBW is based on averaged slope values from three single breaths. We frankly acknowledge that from the current dataset, we cannot definitely explain this phenomenon. In the COPD group, the differences in variability between phase III slope indices of MBW and SIIIDTG are definitely smaller, suggesting that absolute values of phase III slopes have an impact on our calculated variability indices. Technical differences between both tests may contribute as well as differences in phase III slope analysis (automated phase III slope analysis using Spiroware 3.1.6 (pre-set limits 50-95\%) with the option for manual correction in MBW versus automated analysis of SIIIDTG (pre-set limits 60-90\%) with custom software).

The observed variability of the DTG-SBW parameters appeared to be to some extent explained by the variability of the uncontrolled breathing manoeuvre, i.e. flow rate, $V \mathrm{~T}$ or individual lung volume. Therefore, we also considered a volume correction for SIIIDTG (SIIIDTG $\times V$ T) as proposed by SINGER et al. [8]. Due to the fact that healthy and COPD subjects had comparable $V \mathrm{~T}$ around $1 \mathrm{~L}$ (mean $V \mathrm{~T} 1.05 \mathrm{~L}$ in both groups), the correction factor $(V \mathrm{~T})$ was close to 1 and had only minor impact on phase III slope estimates. The 


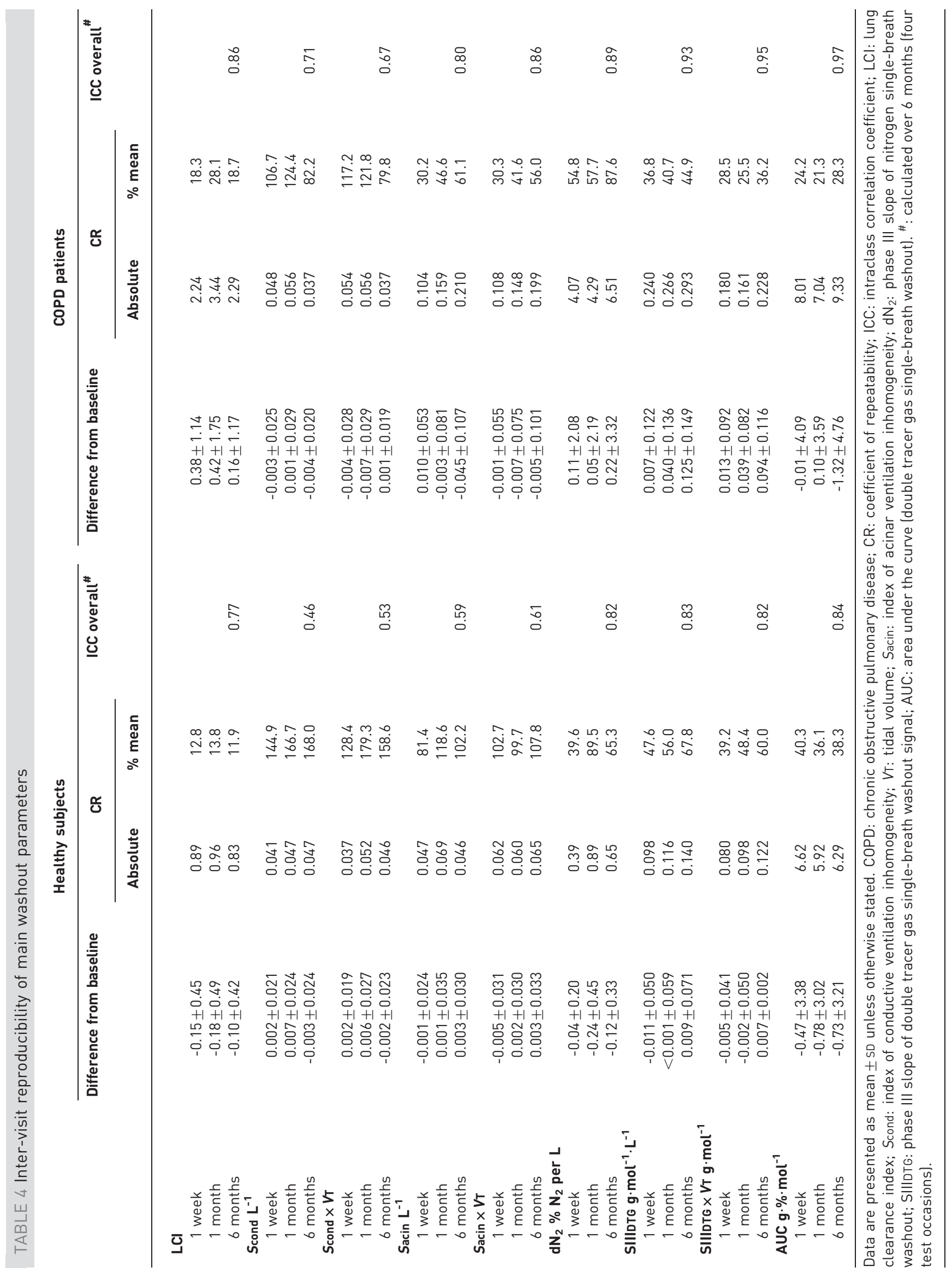



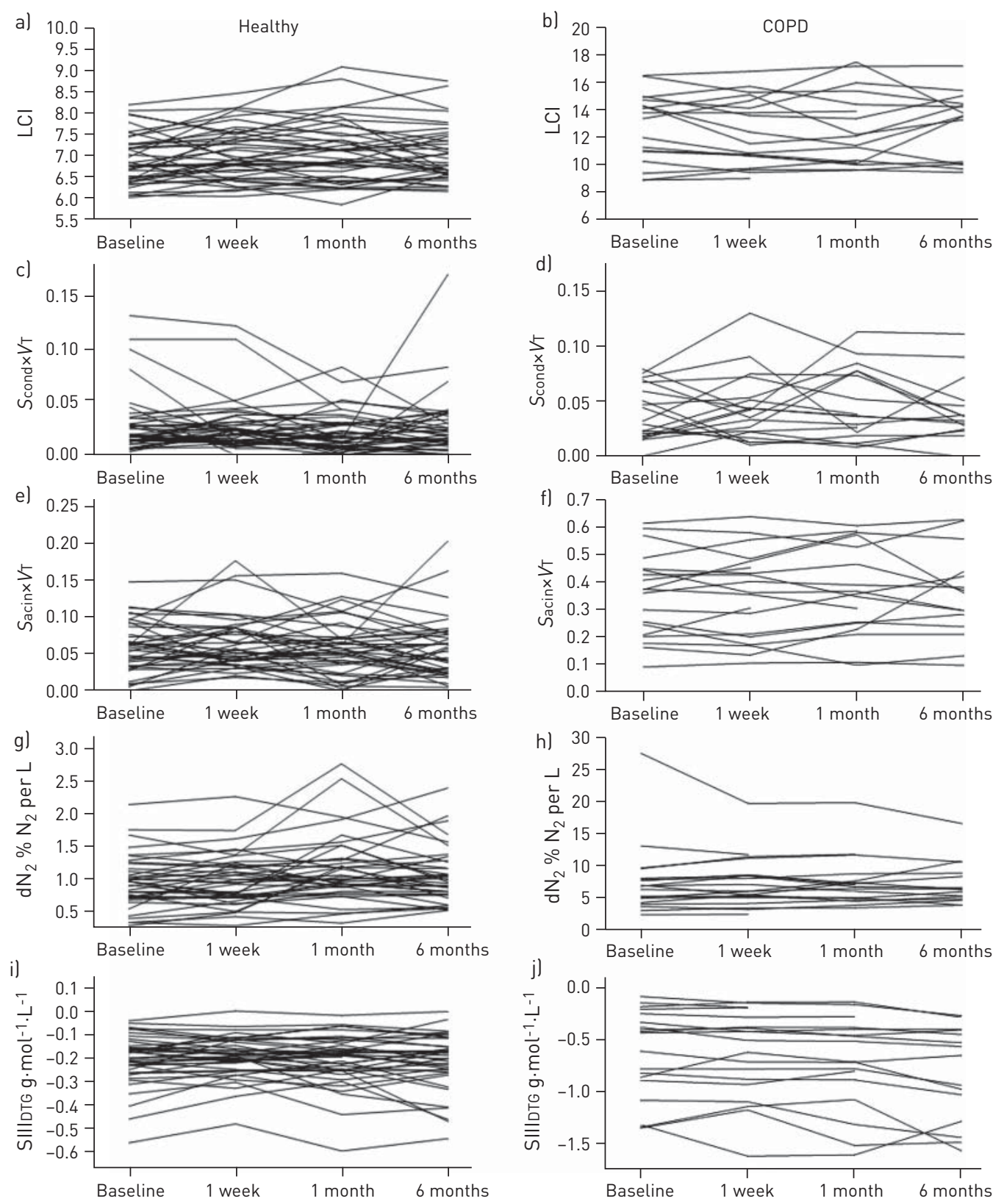

FIGURE 4 Reproducibility of main washout parameters. Means of a, b) lung clearance index (LCI), c, d) conductive ventilation inhomogeneity $($ scond $) \times$ tidal volume $(V T)$, e, f) acinar ventilation inhomogeneity $($ Sacin $) \times V T$, and phase III slope of $\mathrm{g}, \mathrm{h}$ ) nitrogen single-breath washout $\left(\mathrm{dN}_{2-}\right)$ and $\mathrm{i}, \mathrm{j}$ ) dual tracer gas single-breath washout (SIIIDTG) plotted per test occasion and subject in a, c, e, g, i) healthy adults and b, d, f, h, j) chronic obstructive pulmonary disease patients. Intraindividual changes between visits (baseline, 1 week, 1 month and 6 months) are tracked via connecting lines.

variability of SIIIDTG could not be reduced by this volume correction. This is in accordance with the finding that, for all phase III slope indices in our study, the proposed volume correction (multiplication by VT) did not improve reproducibility. Thus, variability of $V \mathrm{~T}$ alone seems not to explain variability of phase III slope indices.

According to our calculated model, the greatest part of the variability in DTG indices is explained by clustering within subjects, not by the breathing pattern itself. Breathing pattern and lung volumes seem to influence DTG-SBW outcomes to some extent in healthy subjects and almost not at all in COPD patients. This reassures us that the different DTG-SBW values observed in COPD are indeed due to the underlying pathology and not due to a difference in breathing pattern. The minor effects of VT, flow or FRC in healthy adults (or of $V \mathrm{~T}$ in COPD) do not seem clinically relevant in the physiological range of tidal flow and $V \mathrm{~T}$ (online supplementary fig. 5). It may be of interest to study this aspect in a more systematic approach performing different breathing manoeuvres in the same subjects. 
The calculation of the coefficient of repeatability provides clinically important data helping to interpret changes in measures of ventilation inhomogeneity over time: in healthy subjects, the coefficient of repeatability for LCI was between $0.83-0.96$. Translated into clinical conditions, this means that the limit of agreement was within 1 unit and a difference $>1$ between two LCI measurements (corresponding to a change over $12-14 \%$ of mean LCI) suggests a significant change of this parameter. Similar data on LCI reproducibility have been reported using the same device in children with cystic fibrosis and healthy controls [35]. In COPD patients, the coefficient of repeatability of ventilation inhomogeneity indices was generally higher due to presumably higher variation of lung function in obstructive lung disease. In clinically stable COPD patients LCI may vary by $2-3$ units (18-28\%) across 6 months.

\section{Limitations}

We acknowledge that we have measured only a small group of COPD patients not matched for age and sex with our healthy subjects. The latter factors could have influenced the comparison of ventilation inhomogeneity indices between groups. Further longitudinal data in larger cohorts are required to adjust for possible age confounding and to better characterise the variability in chronic lung disease.

Due to the clinical and empirical approach of our study, it was not possible to determine the exact location of regional ventilation inhomogeneity leading to altered DTG-SBW parameters in COPD or to provide a definite physiological interpretation of the DTG-SBW signal. Thus the predictive value of DTG indices for structural airway changes needs to be determined.

Another limiting factor of our study is the use of the potent greenhouse gas $\mathrm{SF}_{6}$. Due to shorter test duration and the absence of a vital capacity manoeuvre in the tidal DTG-SBW, the overall consumption of $\mathrm{SF}_{6}$ is considerably lower compared with conventional SBW and MBW tests.

\section{Conclusion}

SIIIDTG as a potential marker of acinar ventilation heterogeneity seems attractive as its natural variability is considerably lower than that of, for example, Scond and Sacin in adults with and without COPD. Change in SIIIDTG $>68 \%$ across 6 months exceeds natural variability (coefficient of repeatability) and thus is clinically relevant, with 95\% probability. The DTG-SBW is feasible and reproducible in healthy subjects as well as in patients with COPD, and can be performed with commercially available hardware. The short test time and the absence of controlled breathing manoeuvres are further advantages. However, custom software is required for analysis. The DTG-SBW may play a future role in early and specific disease detection (e.g. bronchiolitis obliterans and phenotyping of early COPD), monitoring of diseases with small airway dysfunction (e.g. asthma) or as outcome parameters in drug trials to improve small airway function.

\section{Acknowledgements}

The authors would like to thank Mr Rudolf Isler (Eco Medics AG, Duernten, Switzerland) for his valuable technical assistance and Prof. Philipp Latzin (University Children's Hospital, Basel, Switzerland) and Dr Per Gustafsson (Dept of Paediatrics, Central Hospital, Skövde, Sweden) for their scientific support.

\section{References}

1 Robinson PD, Goldman MD, Gustafsson PM. Inert gas washout: theoretical background and clinical utility in respiratory disease. Respiration 2009; 78: 339-355.

2 Robinson P, Latzin P, Verbanck S, et al. Consensus statement for inert gas washout measurement using multiple and single breath tests. Eur Respir J 2013; 41: 507-522.

3 Singer F, Stern G, Thamrin C, et al. Tidal volume single breath washout of two tracer gases-a practical and promising lung function test. PLoS ONE 2011; 6: e17588.

4 Estenne M, Van Muylem A, Knoop C, et al. Detection of obliterative bronchiolitis after lung transplantation by indexes of ventilation distribution. Am J Respir Crit Care Med 2000; 162: 1047-1051.

5 Van Muylem A, De Vuyst P, Yernault JC, et al. Inert gas single-breath washout and structural alteration of respiratory bronchioles. Am Rev Respir Dis 1992; 146: 1167-1172.

6 Van Muylem A, Paiva M, Estenne M. Involvement of peripheral airways during methacholine-induced bronchoconstriction after lung transplantation. Am J Respir Crit Care Med 2001; 164: 1200-1203.

7 Van Muylem A, Baran D. Overall and peripheral inhomogeneity of ventilation in patients with stable cystic fibrosis. Pediatr Pulmonol 2000; 30: 3-9.

8 Singer F, Stern G, Thamrin C, et al. A new double-tracer gas single-breath washout to assess early cystic fibrosis lung disease. Eur Respir J 2013; 41: 339-345.

9 Singer F, Abbas C, Yammine S, et al. Abnormal small airways function in children with mild asthma. Chest 2014; 145: 492-499.

10 Abbas C, Singer F, Yammine S, et al. Treatment response of airway clearance assessed by single-breath washout in children with cystic fibrosis. J Cyst Fibros 2013; 12: 567-574.

11 Global Initiative for Chronic Obstructive Lung Disease. Global Strategy for the Diagnosis, Management and Prevention of COPD. www.goldcopd.org/uploads/users/files/GOLD_Report2014_Feb07.pdf Date last updated: February 7, 2014.

12 Miller MR, Hankinson J, Brusasco V, et al. Standardisation of spirometry. Eur Respir J 2005; 26: 319-338. 
Wanger J, Clausen JL, Coates A, et al. Standardisation of the measurement of lung volumes. Eur Respir J 2005; 26: 511-522.

14 Macintyre N, Crapo RO, Viegi G, et al. Standardisation of the single-breath determination of carbon monoxide uptake in the lung. Eur Respir J 2005; 26: 720-735.

15 Quanjer PH, Stanojevic S, Cole TJ, et al. Multi-ethnic reference values for spirometry for the 3-95-yr age range: the global lung function 2012 equations. Eur Respir J 2012; 40: 1324-1343.

16 Cotes JE, Chinn DJ, Quanjer PH, et al. Standardization of the measurement of transfer factor (diffusing capacity). Eur Respir J 1993; 6: Suppl. 16, 41-52.

17 Verbanck S, Schuermans D, Van Muylem A, et al. Ventilation distribution during histamine provocation. J Appl Physiol 1997; 83: 1907-1916.

18 Aurora P, Kozlowska W, Stocks J. Gas mixing efficiency from birth to adulthood measured by multiple-breath washout. Respir Physiol Neurobiol 2005; 148: 125-139.

19 Chinn S. Statistics in respiratory medicine. 2. Repeatability and method comparison. Thorax 1991; 46: 454-456.

20 Bland JM, Altman DG. Statistical methods for assessing agreement between two methods of clinical measurement. Lancet 1986; 1: 307-310.

21 Sixt R, Bake B, Oxhöj H. The single-breath $\mathrm{N}_{2}$-test and spirometry in healthy non-smoking males. Eur J Respir Dis 1984; 65: 296-304.

22 Hedenström H, Malmberg P, Agarwal K. Reference values for lung function tests in females. Regression equations with smoking variables. Bull Eur Physiopathol Respir 1985; 21: 551-557.

23 Hedenström H, Malmberg P, Fridriksson HV. Reference values for lung function tests in men: regression equations with smoking variables. Ups J Med Sci 1986; 91: 299-310.

24 Houltz B, Green K, Lindblad A, et al. Tidal $\mathrm{N}_{2}$ washout ventilation inhomogeneity indices in a reference population aged 7-70 years. Eur Respir J 2012; 40: Suppl. 56, 694s.

25 Verbanck S, Thompson BR, Schuermans D, et al. Ventilation heterogeneity in the acinar and conductive zones of the normal ageing lung. Thorax 2012; 67: 789-795.

26 Verbanck S, Schuermans D, Van Muylem A, et al. Conductive and acinar lung-zone contributions to ventilation inhomogeneity in COPD. Am J Respir Crit Care Med 1998; 157: 1573-1577.

27 Verbanck S, Schuermans D, Vincken W. Small airways ventilation heterogeneity and hyperinflation in COPD: response to tiotropium bromide. Int J Chron Obstruct Pulmon Dis 2007; 2: 625-634.

28 Cutillo A, Bigler AH, Perondi R, et al. Exercise and distribution of inspired gas in patients with obstructive pulmonary disease. Bull Eur Physiopathol Respir 1981; 17: 891-901.

29 Stuart-Andrews CR, Kelly VJ, Sands SA, et al. Automated detection of the phase III slope during inert gas washout testing. J Appl Physiol 2012; 112: 1073-1081.

30 Van Muylem A, Paiva M, Baran D, et al. Structural change of the acinus during growth assessed by single-breath tracer gas washouts. Pediatr Pulmonol 1996; 22: 230-235.

31 Fuchs SI, Eder J, Ellemunter H, et al. Lung clearance index: normal values, repeatability, and reproducibility in healthy children and adolescents. Pediatr Pulmonol 2009; 44: 1180-1185.

32 Horsley AR, Macleod KA, Robson AG, et al. Effects of cystic fibrosis lung disease on gas mixing indices derived from alveolar slope analysis. Respir Physiol Neurobiol 2008; 162: 197-203.

33 Gonem S, Corkill S, Singapuris A, et al. Between-visit variability of small airway obstruction markers in patients with asthma. Eur Respir J 2014; 44: 242-244.

34 Downie SR, Salome CM, Verbanck S, et al. Ventilation heterogeneity is a major determinant of airway hyperresponsiveness in asthma, independent of airway inflammation. Thorax 2007; 62: 684-689.

35 Singer F, Kieninger E, Abbas C, et al. Practicability of nitrogen multiple-breath washout measurements in a pediatric cystic fibrosis outpatient setting. Pediatr Pulmonol 2013; 48: 739-746. 